\title{
ANÁLISIS DE LOS PERFILES DE CORTA Y LARGA DURACIÓN DE LOS TURISTAS DE OCIO DE BARCELONA
}

\author{
Jorge Moll de Alba \\ Lluís Prats \\ Lluís Coromina \\ Universidad de Girona
}

\section{RESUMEN}

Este artículo analiza el efecto de la duración de la estancia en relación a los conceptos de motivación, satisfacción, imagen, repetitividad y recomendación. El modelo teórico se basa en la literatura y utiliza una metodología de ecuaciones estructurales a través de un análisis multigrupo. El tamaño de la muestra es de 10,953 encuestas a turistas. Los resultados confirman la mayoría de las hipótesis. Por ejemplo, la imagen tiene un efecto en la repetitividad, recomendación y satisfacción. Además, se encuentran diferencias en los efectos de las variables según los turistas de short y long break.

Palabras clave: duración de la estancia; factores push; factores pull; modelo de ecuaciones estructurales; análisis multigrupo.

\section{Analysis of short and long break profiles of leisure tourists in Barcelona}

\section{ABSTRACT}

This article analyses the effect of the length of stay in relation to the constructs of motivation, satisfaction, image, repetition and recommendation. The theoretical model is based on the literature and uses structural equation modelling with multi-group analysis. The size of the sample is 10,953 interviews to tourists. The results confirm most of the hypotheses. For

Recibido: 1 de septiembre de 2015

Devuelto para su revisión: 29 de enero de 2016

Aceptado: 5 de febrero de 2016

Facultat de Turisme. P1. Josep Ferrater i Móra, 1. 17071 GIRONA (España). E-mail: jordi.moll@ud.edu lluis.prats@udg.edu 1luis.coromina@udg.edu 
example, image has an effect on tourist's repeat visitation, recommendation and satisfaction. Furthermore, differences are found among the effects of the variables according to short and long break tourists.

Keywords: length of stay; push factors; pull factors; structural equation modelling; multi-group analysis.

\section{INTRODUCCIÓN}

El incremento del número de turistas y la consolidación de los viajes como un fenómeno global, genera un desarrollo desigual de los destinos y de los diferentes grupos de viajeros. Desde esa perspectiva, dos tendencias significativas de los últimos años han sido, el crecimiento de la capacidad de atracción de los centros urbanos, con los denominados city break (Dunne, Flanagan y Buckley, 2011), y la reducción de la estancia de los turistas en los destinos (Alegre y Pou, 2006). Entre los factores que han contribuido a este incremento de la actividad turística en Europa se pueden destacar, el fenómeno de los viajes de low cost, el aumento del número de viajes y en especial de aquellos viajes cortos o short break, el cambio de percepción de las ciudades como destinos turísticos atractivos, e Internet como herramienta de información, planificación y organización de los viajes, entre otros (Dunne, Flanagan y Buckley, 2010).

También se debe tener en cuenta que un importante número de ciudades europeas han ido sustituyendo las actividades industriales, por servicios avanzados y turismo (Sassen, 2007) y desarrollando estrategias de ciudad basadas o condicionadas por la actividad turística. Barcelona no ha sido ajena a este fenómeno. La terciarización de su economía y la transformación de la ciudad se debe, en parte, al fenómeno del turismo (Casellas, Dot Jutgla y Pallares-Barbera, 2010). Los beneficios económicos que genera el turismo, el acortamiento del tiempo de viaje y el aumento de los viajes, han intensificado la competencia entre ciudades. Por lo que los destinos han incrementado su interés por conocer sus mercados objetivos (Baloglu y McCleary, 1999).

La mejora en el conocimiento de los mercados y consumidores, pone de relevancia cambios substanciales del consumidor turístico que obligan a aquellos destinos que quieren ser competitivos, a adoptar estrategias de supersegmentación, para dar respuesta de manera personalizada a las necesidades de los consumidores (Raj, 2007). Los criterios de segmentación utilizados varían, según el destino, el mercado o el posicionamiento deseado (Buhalis, 2001), y las características principales de los segmentos son múltiples y no afectan al consumo turístico por igual. Así pues, uno de los ámbitos de investigación sobre los que se ha profundizado es el proceso de toma de decisión del consumidor turístico, y de todas las características investigadas, la duración de la estancia destaca como especialmente relevante para la gestión de los destinos turísticos (Martínez-Garcia y Raya, 2008). Además, la duración de la estancia, como variable clave de los turistas permite definir segmentos de viajeros. Los dos grupos utilizados para el análisis en este artículo son: los viajeros de corta duración o short breakers y los de larga duración o long breakers. 
En general, los short break son reconocidos como un tipo diferenciado de viaje, aunque no existe amplio consenso. La definición más aceptada destaca una estancia menor a 4 noches, así como la motivación no profesional (Murphy, Niininen y Sanders, 2010), enfoques que serán usados en este artículo.

Este estudio realiza un análisis del turismo de la ciudad de Barcelona, utilizando modelos de ecuaciones estructurales, dando especial atención a la duración de la estancia y al efecto moderador que ésta tiene sobre el destino turístico.

El presente artículo está dividido en diferentes apartados. En la siguiente sección se revisan los cinco constructos y el efecto de la duración de la estancia. A continuación, se introduce el modelo y la metodología utilizada en la investigación, siguiendo los resultados obtenidos y su interpretación. Finalmente, se describen las conclusiones, así como las limitaciones de este trabajo.

\section{REVISIÓN DE LA LITERATURA}

\subsection{Motivación}

La investigación sobre la motivación de los turistas ha permitido indagar el por qué viajan las personas, y a su vez, estudiar el comportamiento de los viajeros en el destino. De acuerdo con Crompton (1979), en los individuos que viajan por placer, se pueden distinguir dos tipos de motivos, aquellos motivos personales internos y propios del viajero, y aquellos motivos relacionados con el destino y su producto.

Para Yuan y Mcdonald (1990), las personas viajan empujadas por sus fuerzas internas y arrastradas por los atributos externos del destino. Las fuerzas internas, denominadas factores push, son intangibles o intrínsecas de los deseos individuales de los viajeros. Los atributos externos o factores pull son los que surgen como resultado de la capacidad de atracción de un destino. Estos últimos incluyen recursos intangibles, percepciones y expectativas, de los cuales destacamos diez (1) el entretenimiento nocturno, (2) las actividades al aire libre, (3) la cultura diferente a la propia, (4) la naturaleza, (5) los paisajes/escenarios excepcionales, (6) los restaurantes de alta calidad, (7) lugares históricos, (8) museos y galerías de arte, (9) tiempo fiable, (10) variedad de excursiones guiadas, entre otros (Baloglu y Uysal, 1996). Mientras que los factores push, destacamos doce, (1) el deseo de escapar, (2) descansar, (3) relajarse, (4) prestigio, (5) salud, (6) experimentar con la familia junta, (7) experiencia cultural, (8) estar físicamente activos, (9) experimentar formas de vida nuevas y diferentes, (10) darse un capricho o lujo, entre otros (Baloglu y Uysal, 1996; Crompton, 1979; Uysal y Jurowski, 1994).

Baloglu y Uysal (1996) consideran probado que existe relación entre los atributos pull y los motivos de viaje push. Además, diferentes investigaciones plantean que la motivación se puede explicar con factores push y pull, y se centran en la naturaleza multimotivacional de la decisión de viajar para estudiar el comportamiento del turista (Dunne et al., 2011; Iso-Ahola y Allen, 1982; Yuan y Mcdonald, 1990). Ahora bien, se deben aceptar limitaciones en el conocimiento de la motivación y comportamiento de los turistas, como por ejemplo que la motivación mayoritariamente ha sido estudiada en el mundo occidental, que la nacionalidad del turista se ha utilizado como único sustituto para investigar las diferencias culturales en la motivación de viaje, entre otros (Li y Cai, 2011). 
En un entorno competitivo como el actual, resulta de especial interés para la gestión turística profundizar en los factores de motivación de los turistas, ya que ayudan a identificar los atributos que deben ser promocionados (Kozak, 2001), y a concentrarse en segmentos concretos (Martínez-Garcia y Raya, 2008), como por ejemplo, aquellos turistas con más tiempo para viajar o con un mayor gasto turístico. También resulta importante conocer la relación de la motivación con otros elementos como la satisfacción o la repetitividad. Yoon y Uysal (2005) probaron la relación causal entre motivación y satisfacción, mientras que Forgas-Coll, Palau-Saumell, Sánchez-García y CallarisaFiol (2012) corroboraron la relación causal entre motivación y lealtad. En base a estos estudios se plantean las siguientes hipótesis:

H1: Existe una relación positiva entre motivación y satisfacción. El efecto de esta relación será diferente según la duración de la estancia

H2: Existe una relación positiva entre motivación y repetitividad. El efecto de esta relación será diferente según la duración de la estancia

\subsection{Satisfacción}

La satisfacción turística es uno de los elementos más importantes para el éxito del marketing de los destinos, e influencia tanto la selección del destino, como los productos y servicios utilizados y la decisión de volver (Kozak y Rimmington, 2000).

En general, las teorías más comunes sobre la satisfacción parten de la relación entre expectativa-desconfirmación. La satisfacción se puede definir como aquello que hacen los consumidores para llegar a estar satisfechos. El modelo aportado por Oliver (1980), determina que los consumidores desarrollan expectativas sobre el producto previos a la compra. Consecuentemente, el consumidor compara el resultado con las expectativas. Si el resultado es mejor que las expectativas, la desconfirmación es positiva y produce una alta satisfacción, y estarán predispuestos a comprar el producto de nuevo. Oliver y Swan (1989) en la teoría de la equidad determinan que la satisfacción del consumidor puede ser vista como una relación entre el coste, de lo que el consumidor gasta, y la recompensa, que anticipa.

En esta revisión de la literatura cabe destacar algunos trabajos que han demostrado las relaciones entre satisfacción y otros constructos como precio, imagen, lealtad o los atributos del destino (Campo y Yagüe, 2008; Forgas-Coll et al., 2012; Bernini y Cagnone, 2014), así como los recientes estudios que incluyen elementos experienciales como las emociones en la investigación de la satisfacción (Lin, Kerstetter, Nawijn y Mitas, 2014; Charterina y Aparicio, 2015).

En este caso de estudio, se considera la satisfacción en términos idénticos a la satisfacción general (Oliver, 1980). La satisfacción es un estado temporal que se produce tras el consumo y que refleja como el producto o servicio ha cumplido su propósito (Oliver, 1999).

Existen evidencias empíricas sobre que la satisfacción es un antecedente de la lealtad (Oliver, 1999; Oppermann, 2000). En consecuencia, si el nivel de satisfacción es alto, los niveles de lealtad también serán altos. El grado de lealtad se refleja en su 
intención de volver a visitar el destino, repetitividad, y su intención de recomendar, recomendación (Oppermann, 2000). Un turista satisfecho es más probable que tenga intención de volver al destino (Martínez-Ruiz, Garau-Vadell y Campo-Martínez, 2010) y que esté más dispuesto a recomendarlo (Yoon y Uysal, 2005). Por lo que se propone la siguiente hipótesis:

H3: Existe una relación positiva entre satisfacción y repetitividad. El efecto de esta relación será diferente según la duración de la estancia

H4: Existe una relación positiva entre satisfacción y recomendación. El efecto de esta relación será diferente según la duración de la estancia

\subsection{Imagen}

La revisión bibliográfica sobre la imagen turística evidencia la multidisciplinariedad desde la que ha sido estudiada (Gallarza, Gil-Saura y Calderó-García, 2002). Desde la perspectiva de la definición conceptual, el inicio de la imagen turística parte de los trabajos de Hunt en 1971. Desde entonces, numerosos autores han desarrollado sus trabajos aportando conocimiento sobre el tema. Hunt (1971) entiende la imagen turística como las impresiones que una persona o personas tienen de un sitio en el cual no residen. Fakeye y Crompton (1991) destacan en su definición el concepto de imagen general, mientras que para Kotler, Haider y Rein (1994) la imagen de un destino es una suma de elementos como creencias, ideas, e impresiones, que una persona tiene sobre un lugar.

De las diferentes líneas de investigación sobre imagen, cabe destacar las relacionadas con el proceso de formación de imagen y las diferentes tipologías de imagen propuestas. La mayoría de autores están de acuerdo en considerar la imagen como una construcción mental. Gartner (1994) introdujo tres componentes relacionados en la formación de la imagen: el cognitivo, afectivo y conativo. Baloglu y McCleary (1999) dividen la imagen en variables cognitivas y en variables afectivas; generando lo que denominan una imagen global. Stern y Krakover (1993) en su modelo de formación de la imagen de una ciudad, también confirman que las variables perceptivas / cognitivas y afectivas, generan la imagen global de un ámbito urbano, formando una imagen compuesta o general de la ciudad.

En este artículo se considera la imagen del destino en términos de imagen global o general de la ciudad, considerando las interacciones entre los distintos tipos de imagen: la emitida por parte de la industria local y la percibida por parte del consumidor (Govers y Go, 2004). También es necesario remarcar que la presente investigación se centra en la imagen percibida in situ (Galí Espelt y Donaire Benito, 2005), mediante encuestas realizadas a turistas en el destino.

Otro elemento sobresaliente de investigaciones anteriores que debemos tener presente es que la imagen del destino afecta las percepciones y el comportamiento del turista. Mientras que Camprubí, Guia y Comas (2009) consideran la imagen turística como uno de los elementos importantes de competitividad de los destinos turísticos; para Gartner (1989), Crompton y Ankomah (1993), Sirakaya-Turk, Sonmez y Choi (2001), Chi y Qu (2008), la imagen influencia a los turistas en la selección del destino. De esa 
manera, los destinos con mejor imagen serán mejor considerados en los procesos de decisión (Chi y Qu, 2008). Según Mohammad y Som (2010) la motivación está asociada con la competitividad del destino y a la imagen del mismo. Por lo que podemos plantear también la siguiente hipótesis:

H5: Existe una relación positiva entre motivación e imagen del destino. El efecto de esta relación será diferente según la duración de la estancia

Por su parte, Lupton y Court (1997) y Martínez-Ruiz et al. (2010) demuestran empíricamente que la imagen turística del destino afecta positivamente la intención de volver a visitarlo en el futuro. Bigné Alcañiz, Sánchez y Sánchez (2001), Phillips, Wolfe, Hodur y Leistritz (2013) y Zhang, Fu, Cai y Lu (2014) prueban el efecto de la imagen del destino en la recomendación. Para comprobar la relación entre la imagen del destino, la repetitividad y la recomendación, se proponen las siguientes hipótesis de trabajo:

H6: Existe una relación positiva entre imagen del destino y repetitividad. El efecto de esta relación será diferente según la duración de la estancia

H7: Existe una relación positiva entre imagen del destino y recomendación. El efecto de esta relación será diferente según la duración de la estancia

Para Chon (1991), Fakeye y Crompton (1991), la creación de una imagen positiva del destino puede mejorar la satisfacción del turista. La mejora en las necesidades de los turistas se asocia a una mejora en su experiencia, y esta produce una realidad positiva. Este último punto permite generar la siguiente hipótesis, basada en si la estancia del turista en el destino ha generado una alta satisfacción, el consumidor generará un mensaje e imagen positiva del destino.

H8: Existe una relación positiva entre imagen del destino y satisfacción. El efecto de esta relación será diferente según la duración de la estancia.

\subsection{Repetitividad}

El concepto de repetitividad forma parte del comportamiento post-venta del consumidor. Martínez-Ruiz et al. (2010) profundizan en la repetitividad desde dos perspectivas muy diferentes, la experiencia previa y la probabilidad de volver. Kozak (2001) por ejemplo, compara las experiencias de los turistas de acuerdo a si son, o no, repetidores. Otros investigadores han concentrado su trabajo en los factores que influencian las visitas repetitivas a un destino (Kozak, 2001; Martínez-Ruiz et al., 2010; Meleddu, Paci y Pulina, 2015). Factores positivos como el contacto con la naturaleza, la limpieza o la accesibilidad, o negativos como la carestía de precios, el desarrollo excesivo y la sobreconstrucción, entre otros, son elementos motivacionales y de satisfacción que condicionan la repetitividad (Alegre y Garau, 2010). En el caso de Som, Marzuki, Yousefi y AbuKhalifeh (2012) se destacan la imagen del destino, la relajación y la diversión como principales atributos del destino que condicionan la repetitividad. 
Otra línea de investigación sobre el tema de la repetitividad está directamente relacionada con el concepto de lealtad. Oliver (1999) define el concepto de lealtad como el compromiso implicado en la transición de una predisposición favorable, lealtad afectiva, a un compromiso de compra repetida, lealtad conativa, como paso previo a la acción de compra. En esta definición el elemento trascendental es la repetitividad de la compra, y que éste es uno de los indicadores más importantes para medir el resultado de las estrategias de marketing (Flavian, Martínez y Polo, 2001).

Del estudio de la repetitividad y lealtad de los destinos destaca el esfuerzo realizado para probar las relaciones con otros conceptos como la satisfacción, imagen y motivación. La satisfacción es un determinante de la lealtad para Chen y Phou (2013) y Yoon y Uysal (2005). Chi y Qu (2008) prueban relaciones positivas y directas entre satisfacción y lealtad y Kozak, Bigne y Andreu (2005) que la satisfacción y el número de visitas anteriores influencia las intenciones de realizar futuras visitas. Para Moniz (2012), la repetición en turismo se sustenta en la satisfacción general del destino y la satisfacción específica con atributos del destino. Además, tal como se ha mencionado en apartados anteriores Lupton y Court (1997), Martínez-Ruiz et al. (2010), Da Costa Mendes, Do Valle, Guerreiro y Silva (2010), demuestran relaciones entre la imagen turística y la intención de volver a visitar el destino por parte del turista. Mientras que Yoon y Uysal (2005) prueban la relación entre motivación y lealtad.

Por último, cabe destacar que Oppermann (2000) prueba que existen evidencias de que los turistas repetidores son más propensos a transmitir su experiencia positiva recomendando el destino y André Romero (1999) introduce la variable de duración de la estancia, como una de las variables explicativas de su modelo que permite estimar la probabilidad de que un individuo repita su visita. Este último punto nos permite generar la siguiente hipótesis basada en la duración de la estancia.

H9: Existe una relación positiva entre repetitividad y recomendación. El efecto de esta relación será diferente según la duración de la estancia

Como resumen se puede decir que la repetitividad forma parte de un concepto más amplio, la lealtad. La lealtad obliga a los destinos a adoptar estrategias innovadoras, para estimular la repetición del turista. De esta manera, se pone de relieve la importancia de la lealtad a largo plazo en las estrategias de los destinos (Fyall, Callod y Edwards, 2003).

\subsection{Recomendación}

La recomendación forma parte del comportamiento posterior a la experiencia de compra y la utilización del bien o servicio por parte del consumidor. En el ámbito turístico la recomendación, ha atraído la atención de investigadores, debido principalmente a que se puede considerar que es uno de los mejores indicadores de lealtad de los turistas.

En este caso de estudio, se considera la recomendación como la intención que tiene un turista por transmitir su experiencia de viaje, una vez haya acabado su estancia en el destino. Dentro de la recomendación se debe destacar la importancia de la comu- 
nicación boca-oreja, WOM, definida como una comunicación informal de persona a persona entre un comunicador no comercial y un receptor, con respecto a una marca, un producto, una organización o un servicio (Anderson, 1998). La investigación sobre la comunicación WOM, no sólo se ha centrado en la recomendación positiva, si no que investigadores como Cheng, Lam y Hsu (2006), han profundizado en la comunicación WOM negativa.

De los estudios sobre recomendación se debe mencionar la relevancia de la investigación para probar las relaciones de ésta, con otros constructos. Investigadores como Kozak (2001), Kozak y Rimmington (2000), Yoon y Uysal (2005), consideran probado que la satisfacción es un indicador de la intención de recomendar de los turistas a otras personas. Por lo que un turista satisfecho es más probable que repita y recomiende su experiencia positiva del destino a familiares y amigos (Chi y Qu, 2008). Este comportamiento, la recomendación, es destacado por Yoon y Uysal (2005) como elemento crítico para el marketing turístico.

\subsection{El efecto de la duración de la estancia: short break y long break}

En el contexto actual del turismo, existen evidencias de una reducción progresiva de la duración de la estancia de los viajes turísticos. Entre las diferentes variables estudiadas sobre el comportamiento de los turistas y la demanda turística, la duración de la estancia es, sin duda alguna, una de las claves de la gestión turística de destinos (Martínez-Garcia y Raya, 2008).

En general, la duración de la estancia ha sido estudiada desde dos perspectivas diferentes. La primera es desde la descripción de los perfiles de los turistas y los tipos de viajes de acuerdo a la duración de su estancia (Molina Collado, 2007; Oppermann, 1995). La segunda es desde una perspectiva econométrica, aplicando diferentes modelos para explicar y predecir en el tiempo esta característica clave de la demanda. Barros, Correia y Crouch (2008) analizaron la duración de la estancia de los turistas con modelos de supervivencia y logístico. Peypoch, Randriamboarison, Rasoamananjara y Solonandrasana (2012) emplean el multivariate fractional polynomial para medir la longitud de la estancia de los turistas en el destino. Martínez-Garcia y Raya (2008) realizaron su análisis con modelos de supervivencia de distribución exponencial-logarítmica y de regresión de Cox. Alegre y Pou (2006) utilizan la elección discreta / modelo continuo. Para ellos, el tiempo que el turista decide quedarse es una característica básica de las vacaciones. Mientras que Assaf, Pestana Barros y Gil-Alana (2010) usan datos de series temporales simultáneamente con fractional integration y seasonal autoregressions. Alén et al. (2014) utilizan una distribución binomial negativa para determinar los factores que influencian la duración de la estancia y Ferrer-Rosell, Martínez-Garcia y Coenders (2014) utilizan logit ordernado con efecto moderador de la duración de la estancia. André Romero (1999) plantea una regresión binomial, en el que la duración de la estancia es una de las variables explicativas del modelo, que permite estimar la probabilidad de que un individuo repita su visita, y un modelo de gasto con segmentación mediante regresión.

En este trabajo de investigación, se considera la duración de la estancia como base para segmentar la demanda (Molina Collado, 2007), de acuerdo con el número de días que 
permanece el turista en el destino. Tomando esa variable como criterio de segmentación, se pueden diferenciar dos grupos, los viajes de corta duración o short break y los viajes de larga duración o long break.

Los short break es un segmento limitado en cuanto a conocimiento, mientras que el caso de los long break no ha sido específicamente abordado, considerándose simplemente lo opuesto a los short break.

Tsiotsou y Vasioti (2006) destacan que los visitantes de corta duración no han sido estudiados adecuadamente. Davies (1990) se plantea la dificultad de definir los short break debido a la incapacidad de la industria turística de acordar una definición consensuada. Davies considera que los short break son viajes cortos que incluyen: prestación de servicios de alojamiento, transporte y entradas que dependen del objeto del viaje. Por su parte Edgar (1997) aborda los viajes de short break desde la perspectiva de mercado, y los considera un mercado clave para que los hoteles incrementen su rentabilidad mediante técnicas de Yield Management; y Buhalis (2001) en su aproximación a la demanda turística define las "S"s del turismo convencional de sol y playa (sea, sun, sand, sex, sangría) y del turismo urbano (short break, sightseing, shopping, shows, etc.), considerando el short break como una especialización del turismo urbano.

Entre los agentes turísticos existe cierta confusión entre los viajes de short break y los denominados city break. Si bien (Dunne et al., 2010) consideran necesario tener claro que city break y short break son segmentos distintos. Para este artículo ambos son coincidentes al haberse analizado el caso de los turistas de la ciudad de Barcelona. Los city break son viajes que tienen una naturaleza de ocio y se circunscriben al ámbito urbano, sin tener en consideración la duración de la estancia del viajero, aunque habitualmente suelen ser viajes de menos de 3 noches (Dunne et al., 2010). Otra confusión es la utilización del término short break para referirse a visitas de día o el segmento de excursionista, es decir sin pernoctación (Downward y Lumsdon, 2003; Tsiotsou y Vasioti, 2006).

En este artículo se adopta la definición del Sustainable Tourism Cooperative Research Centre (STCRC). Un short break es "un viaje no profesional de entre una y cuatro noches fuera de su lugar habitual de residencia" (Murphy et al., 2010). Esta misma fuente recomienda "acotar esta definición a los viajes entre dos y cuatro noches" para facilitar su identificación, la cual ha sido adoptada en este artículo.

En los estudios de la STCRC se determina que, sólo en Australia, el 54\% de los residentes que viajaron en el año 2010, realizaron un viaje de short break. Pike (2009) identifica que Australia ha mantenido el posicionamiento de short break, al menos durante un periodo no inferior a cuatro años. Y lo que es aún más importante, incluso en momentos de recesión económica generalizada, este mercado continúa expandiéndose, no sólo en Australia, sino a nivel global (Murphy et al., 2010).

La globalización y la evolución de los estilos de vida han provocado el surgimiento de nuevos comportamientos para viajar, nuevas actividades y nuevas formas de turismo como el turismo urbano y el de estancias cortas o short break. Los short break suelen estar estrechamente relacionados con el turismo y las actividades urbanas, incluir visitas puntuales y generalizadas (sightseeing), compras en áreas comerciales (shopping), una concentración de actividades de ocio y espectáculos (shows) y una duración de la estancia entre 2 y 4 noches. 


\subsection{Modelo estructural propuesto}

En la Figura 1 se representa el modelo teórico causal propuesto. Los componentes del modelo corresponden con las hipótesis anteriormente mencionadas. El constructo motivación se mide a través de una variable dicotómica motivación, donde $0=$ factores push y 1 = factores pull. Estudios anteriores probaban la influencia de la motivación en la satisfacción (Oliver, 1999), la motivación en la imagen (Mohammad y Som, 2010), y la imagen en la satisfacción (Chon, 1991). Por lo tanto, el modelo causal propuesto, analiza las relaciones entre motivación, satisfacción, imagen, repetitividad y recomendación con la principal contribución de usar la variable la duración de la estancia (short break y long break) como discriminatoria de los turistas en el modelo multigrupo.

\section{Figura 1 \\ MODELO ESTRUCTURAL PROPUESTO}

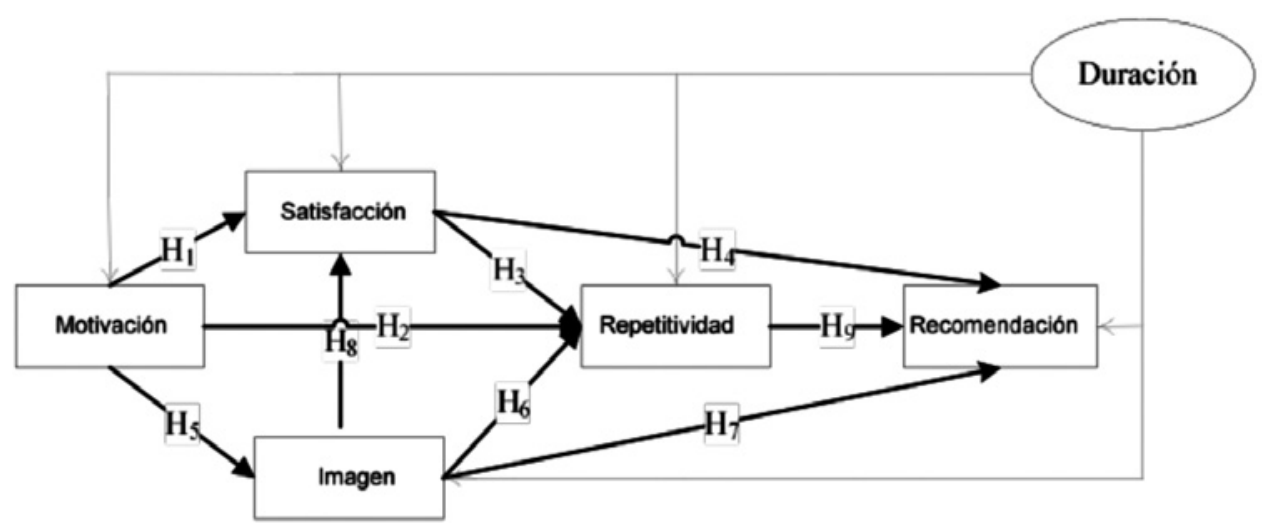

\section{MÉTODOS}

\subsection{Caso de investigación}

Para testar el modelo teórico se han seleccionado los turistas que visitan la ciudad de Barcelona por diversos motivos. En primer lugar, Barcelona se ha convertido en una de las ciudades turísticas más atractivas de Europa (Valls et al., 2013). Los más de 7.8 millones de turistas que pernoctaron en los hoteles de la ciudad en el año 2014, generaron más de 17 millones de pernoctaciones (Turisme de Barcelona, 2014). Estas cifras, sitúan a Barcelona en el top 6 del ranking de ciudades turísticas europeas (ECM, 2013). Dentro de las diferentes tipologías y segmentos de demanda de la ciudad, resulta destacable, entre otros, los 2.4 millones de cruceristas, los 579 mil delegados participantes en eventos de turismo MICE (Turisme de Barcelona, 2014) o los más de 12 millones de excursionistas que visitaron la ciudad (Oficina PET BCN2015, 2009). 
En segundo lugar, el éxito de la ciudad, gracias a la combinación de estrategias culturales, de regeneración urbana y de gobernanza, han consolidado un modelo exitoso denominado Modelo Barcelona (Degen y García, 2012). En ese modelo la creación de imagen y el turismo han sido elementos clave, así como grandes eventos como los Juegos Olímpicos de 1992 (Casellas et al., 2010). Ahora bien, de cara al futuro para favorecer la sostenibilidad del turismo en la ciudad, desde las autoridades municipales mediante el "Pla Estratègic de Turisme de la Ciutat de Barcelona 2015", se impulsa el equilibrio en la relación entre residentes y turistas; así como entre el turismo y el resto de actividades económicas (Casellas et al., 2010) y el conocimiento.

Aun así, la dimensión del fenómeno turístico de la ciudad no se corresponde con las investigaciones académicas realizadas. En ese ámbito, es importante destacar el esfuerzo realizado por Turisme de Barcelona y el Pla Estratègic de Turisme de la Ciutat de Barcelona 2010-2015, así como la labor de determinados investigadores como Casellas et al. (2010), Forgas-Coll et al. (2012), Palau-Saumell, Forgas-Coll, Sanchez-Garcia y PratsPlanaguma (2012), Palou Rubio (2011), Valls, Banchini, Falcón y Valls Tuñon (2013), entre otros.

En tercer lugar, el desconocimiento generalizado y la falta de estudios de segmentos concretos de demanda turística con potencialidad, como es el caso de los short break, corroboran la idoneidad de este artículo, y ponen de relieve la importancia de nuevas estrategias, tanto para la DMO, Destination Marketing Organisation, como para el sector privado, priorizando las actuaciones sobre microsegmentos de demanda.

\subsection{Muestra y recopilación de datos}

Para la evaluación del modelo, de las relaciones entre los diferentes constructos y el efecto moderador de la duración de la estancia, se ha partido de la encuesta que realiza Turisme de Barcelona, a los turistas de la ciudad de Barcelona en los años 2009, 2010 y 2011.

La variable discriminatoria que permite llevar a cabo un análisis multigrupo es la duración de la estancia. Un primer grupo denominado short break, donde el número de noches que ha pernoctado o piensa pernoctar el turista en Barcelona está entre 2 y 4 , y un segundo grupo llamado long break, donde el número de noches que ha pernoctado o piensa pernoctar el turista en Barcelona es superior a 4. Se debe tener en cuenta que, siguiendo las definiciones de short break, la muestra analizada se basa en turismo de ocio/vacacional.

La Tabla 1 muestra las variables usadas para estimar el modelo estructural y estudiar las hipótesis planteadas en el modelo. Las medias y desviaciones estándar de la lista de los ítems de la Tabla 3, dividida por short y long break se muestra en la Tabla A1 del Anexo.

Los turistas fueron entrevistados durante el periodo de tiempo comprendido entre los años 2009 y 2011, realizando entrevistas todos los días de la semana, en diferentes horarios y localizaciones de la ciudad (hoteles y puntos de atracción turística dentro del término municipal de Barcelona). La población consistía en todos los visitantes mayores de 14 años que pernoctan en Barcelona menos de 30 días. 


\section{Tabla 1}

VARIABLES UTILIZADAS EN EL MODELO

DE ECUACIONES ESTRUCTURALES

\begin{tabular}{|c|c|c|}
\hline Nombre de la variable & $\begin{array}{l}\text { Escala de } \\
\text { medida }\end{array}$ & Definición \\
\hline "Motivación push/pull" & Dicotómica & $\begin{array}{l}\text { Por qué motivo se ha decidido a venir a } \\
\text { Barcelona } \\
0=\text { factores push } \\
1=\text { factores pull }\end{array}$ \\
\hline $\begin{array}{l}\text { "Imagen percibida" } \\
\text { Amplia oferta de puntos de interés } \\
\text { Tiene una vida cultural muy rica }\end{array}$ & Métrica & $\begin{array}{l}\text { Valorándose escala Likert } \\
1=\text { Totalmente de acuerdo } \\
5=\text { Totalmente en desacuerdo }\end{array}$ \\
\hline "Satisfacción general" & Métrica & $\begin{array}{l}\text { Valorándose escala Likert } \\
1=\text { mínimo } \\
10=\text { máximo }\end{array}$ \\
\hline "Repetitividad" & Métrica & $\begin{array}{l}\text { ¿Cuántas veces ha visitado Barcelona en } \\
\text { los últimos } 10 \text { años? }\end{array}$ \\
\hline "Recomendación" & Métrica & $\begin{array}{l}\text { Escala Likert } \\
1=\text { Seguro que no } \\
5=\text { Seguro que si }\end{array}$ \\
\hline
\end{tabular}

Un total de 10953 turistas tienen una motivación vacacional/ocio, y han estado en Barcelona 2 o más noches. La Tabla 2 muestra la distribución de la muestra para los short break y long break.

Tabla 2

CARACTERÍSTICAS SELECCIONADAS DEL PERFIL DE LA MUESTRA

\begin{tabular}{lrrrrrr}
\hline Características demográficas & $\begin{array}{r}\text { Short } \\
\text { break }\end{array}$ & $(\%)$ & $\begin{array}{r}\text { Long } \\
\text { break }\end{array}$ & $(\%)$ & Total & $(\%)$ \\
\hline Género & & & & & & \\
Hombre & 3599 & 55.04 & 2436 & 55.19 & 6035 & 55.10 \\
Mujer & 2940 & 44.96 & 1978 & 44.81 & 4918 & 44.90 \\
Total & 6539 & 100 & 4414 & 100 & 10953 & 100 \\
\hline Edad & & & & & & \\
15-17 años & 88 & 1.35 & 70 & 1.59 & 158 & 1.44 \\
$18-24$ años & 1112 & 17.01 & 746 & 16.90 & 1858 & 16.96 \\
$25-34$ años & 1811 & 27.70 & 1059 & 23.99 & 2870 & 26.20 \\
35-44 años & 1706 & 26.09 & 1299 & 29.43 & 3005 & 27.44 \\
$45-54$ años & 1171 & 17.91 & 806 & 18.26 & 1977 & 18.05 \\
55-64 años & 498 & 7.62 & 338 & 7.66 & 836 & 7.63 \\
65 o más años & 153 & 2.34 & 96 & 2.17 & 249 & 2.27 \\
Total & 6539 & 100 & 4414 & 100 & 10953 & 100 \\
\hline
\end{tabular}




\begin{tabular}{|c|c|c|c|c|c|c|}
\hline \multicolumn{7}{|l|}{ Ocupación } \\
\hline $\begin{array}{l}\text { Autónomo/ Cuenta propia/ } \\
\text { Agricultor }\end{array}$ & 641 & 9,80 & 527 & 11,94 & 1.168 & 10,66 \\
\hline $\begin{array}{l}\text { Alto ejecutivo/ Alto } \\
\text { funcionario }\end{array}$ & 188 & 2.88 & 138 & 3.13 & 326 & 2.98 \\
\hline Administrativo/ Funcionario & 861 & 13.17 & 379 & 8.59 & 1240 & 11.32 \\
\hline Trabajador especializado & 2176 & 33.28 & 1502 & 34.03 & 3678 & 33.58 \\
\hline Trabajador en otro campo & 1101 & 16.84 & 726 & 16.45 & 1827 & 16.68 \\
\hline Jubilado & 243 & 3.72 & 154 & 3.49 & 397 & 3.62 \\
\hline Ama de casa & 242 & 3.70 & 190 & 4.30 & 432 & 3.94 \\
\hline Estudiante & 897 & 13.72 & 704 & 15.95 & 1601 & 14.62 \\
\hline Parado & 116 & 1.77 & 48 & 1.09 & 164 & 1.50 \\
\hline Otros & 74 & 1.13 & 46 & 1.04 & 120 & 1.10 \\
\hline Total & 6539 & 100.00 & 4414 & 100.00 & 10953 & 100 \\
\hline \multicolumn{7}{|l|}{ Nacionalidad } \\
\hline Española & 788 & 12.05 & 285 & 6.46 & 1073 & 9.80 \\
\hline Francesa & 752 & 11.50 & 352 & 7.97 & 1104 & 10.08 \\
\hline Británica & 734 & 11.22 & 370 & 8.38 & 1104 & 10.08 \\
\hline Italiana & 721 & 11.03 & 422 & 9.56 & 1143 & 10.44 \\
\hline Alemana & 486 & 7.43 & 285 & 6.46 & 771 & 7.04 \\
\hline EEUU & 438 & 6.70 & 463 & 10.49 & 901 & 8.23 \\
\hline Otros & 2620 & 40.07 & 2237 & 50.68 & 4857 & 44.34 \\
\hline Total & 6539 & 100 & 4414 & 100 & 10953 & 100 \\
\hline
\end{tabular}

La Tabla 3 muestra las características del viaje para los turistas de short y long break en la ciudad de Barcelona.

Tabla 3

CARACTERÍSTICAS SELECCIONADAS DEL VIAJE

\begin{tabular}{lrrrrrr}
\hline Características del viaje & $\begin{array}{r}\text { Short } \\
\text { break }\end{array}$ & $(\%)$ & $\begin{array}{r}\text { Long } \\
\text { break }\end{array}$ & $(\%)$ & Total & $(\%)$ \\
\hline Alojamiento & & & & & & \\
Hotel & 3999 & 61.16 & 2218 & 50.25 & 6217 & 56.76 \\
Pensión & 910 & 13.92 & 264 & 5.98 & 1174 & 10.72 \\
Apartamento, casa de alquiler & 282 & 4.31 & 805 & 18.24 & 1087 & 9.92 \\
Albergue, residencia estudiante & 769 & 11.76 & 499 & 11.30 & 1268 & 11.58 \\
Casas de amigos & 544 & 8.32 & 626 & 14.18 & 1170 & 10.68 \\
Cruceros & 34 & 0.52 & 0 & 0.00 & 34 & 0.31 \\
Otros & 1 & 0.02 & 2 & 0.05 & 3 & 0.03 \\
Total & 6539 & 100 & 4414 & 100 & 10953 & 100 \\
\hline
\end{tabular}




\begin{tabular}{lrrrrrr}
\hline $\begin{array}{l}\text { Estancia media } \\
\text { Noches }\end{array}$ & 3.12 & & 7.28 & & & \\
\hline Repetitividad & & & & & & \\
No (primera visita) & 3977 & 61.60 & 2823 & 64.54 & 6800 & 62.79 \\
Sí, 2a visita & 1528 & 23.67 & 838 & 19.16 & 2366 & 21.85 \\
Sí, 3a o más visitas & 951 & 14.73 & 713 & 16.30 & 1664 & 15.36 \\
Total & 6456 & 100.00 & 4374 & 100.00 & 10830 & 100 \\
\hline
\end{tabular}

Los resultados de la Tabla 3 relacionados con las características del viaje señalan aspectos distintivos del segmento de short break, como por ejemplo, muestran una preferencia a alojarse en hoteles. Otra característica distintiva es que el porcentaje de repetidores en los turistas de short break es más elevado.

\subsection{Data análisis}

El análisis de datos se realizó en dos etapas diferentes. Primero, un análisis exploratorio de las variables con SPSS 19. Este análisis fue realizado para identificar la dimensión de la motivación push y pull, satisfacción, imagen, recomendación y repetitividad.

Las relaciones entre motivación, satisfacción, imagen, repetitividad y recomendación fueron empíricamente probadas utilizando el modelo de ecuaciones estructurales (SEM) con Mplus 7.

El análisis multigrupo analiza si el modelo se mantiene para los niveles de la variable moderadora, duración de la estancia, donde los niveles del moderador (short break y long break) representan grupos mutuamente excluyentes e independientes. Se estimó el modelo estructural usando Robusta Máxima Versemblancia (RML - Robust máximum likelihood).

Inicialmente se determinaron los factores push y pull de la variable motivación de viaje de acuerdo a Baloglu y Uysal (1996). La clasificación obtenida se muestra en la Tabla 4, donde los factores se relacionan en orden decreciente de prevalencia.

El descanso y las vacaciones son el motivo push más importante de los turistas de Barcelona. Este motivo de viaje puede estar relacionado con los motivos de evadirse o escaparse. Iso-Ahola y Allen (1982) conceptualiza esta motivación con el concepto "avoidance", realizar vacaciones para escaparse de algo o de alguien. Los resultados también demuestran que los viajeros están cansados de sus labores habituales y que necesitan descansar/relajarse realizando actividades diferentes según sus propios intereses, visitando monumentos, asistiendo a espectáculos, etc.

El segundo y tercer motivos push más populares para realizar un viaje está relacionado con el deseo y las ganas de conocer la ciudad y España. Crompton (1979) considera que, los motivos culturales y de conocimiento son grandes elementos motrices que llevan a los turistas a viajar. Del resto de factores push, cabe mencionar aquellos relacionados con "el fortalecimiento de las relaciones de parentesco". Diversos participantes confirman que el motivo para realizar un viaje de short break es servir como medio para reunirse con los miembros de su familia o amigos, que por diversos motivos viven habitualmente separados. 
Tabla 4

FACTORES DE MOTIVACIÓN PUSH Y PULL

\begin{tabular}{lrlr}
\hline Factores push & $(\%)$ & Factores pull & $(\%)$ \\
\hline Vacaciones/ Descanso & 55.14 & Gusta la ciudad & 36.92 \\
Ganas de conocer la ciudad & 12.74 & Arquitectura y sus monumentos & 16.02 \\
Para visitar España & 5.12 & Buenos precios para viajar/oferta low cost & 7.69 \\
Amigos que viven & 4.54 & Mucha oferta para visitar/cosas para ver & 6.76 \\
Familiares que viven & 4.04 & Gaudí & 6.23 \\
Estudios & 2.53 & Es interesante & 4.25 \\
Por la fama y el nombre que tiene & 2.28 & Cultura & 4.14 \\
Volver a visitar la ciudad & 1.60 & Ambiente & 3.84 \\
Por la diversión & 1.49 & Por clima & 3.79 \\
Nunca ha visitado Barcelona & 1.39 & Ver partido de futbol & 2.85 \\
Acompañar padres & 1.36 & Playa & 1.86 \\
Se la han recomendado & 1.36 & Cosmopolita & 1.40 \\
Acompaña marido/ mujer & 1.35 & Por la historia & 1.22 \\
Visitar hijo/ hija & 1.12 & Tiendas y comercio & 0.99 \\
Visitar la pareja & 0.97 & Ciudad gran & 0.99 \\
Visita/ Tratamiento médico & 0.84 & Es diferente & 0.29 \\
Viaje de novios & 0.66 & Feria & 0.29 \\
Viaje Regalo & 0.63 & Gastronomía & 0.23 \\
Viaje final de curso/ carrera & 0.51 & Ópera & 0.23 \\
Familiar enfermo & 0.34 & & \\
\hline & & &
\end{tabular}

Entre los principales motivadores pull cabe destacar el factor, "Barcelona gusta" como el más importante, seguido de la "Arquitectura y sus monumentos", "Mucha oferta para visitar/cosas para ver", "Gaudí" y la "Cultura". Estos últimos están muy ligados con el destino urbano de la ciudad de Barcelona. El factor "Buenos precios para viajar/oferta low cost" está directamente relacionado con el fenómeno de las compañías aéreas de bajo coste. Estas compañías han incrementado la accesibilidad a los viajes por parte de la población y han favorecido el crecimiento del número de viajeros especialmente en Europa, generando un auténtico cambio en el mercado turístico. Otro motivo digno de mención, en el caso de los viajes a Barcelona, es el atractivo de actividades programadas en la ciudad como "Ver partido de fútbol", "Opera", entre otros. Un número importante de viajes incluyen en sus visitas y son motivos de sus viajes, el fútbol, conciertos, fiestas, exhibiciones y salones.

El último grupo de factores pull importantes está formado por elementos físicos de la ciudad como la playa, las tiendas y el comercio o la gastronomía. Estos factores 
se complementan con elementos intangibles como "el ambiente", "cosmopolita", "es diferente", etc. Los resultados demuestran la variedad y naturaleza multimotivacionales de la ciudad de Barcelona.

Los factores de motivación push y pull, se recodificaron en una variable dicotómica que denominamos motivación, donde los factores push, toman el valor 0 , y los factores pull, toman el valor 1. Los resultados de la tabla de contingencia entre la elección de motivos push y pull con la duración de la estancia (short y long break) muestran que hay una relación estadísticamente significativa $\left(\chi^{2}=5.871,1\right.$ grado de libertad, p-valor=.015) entre ambas. Es decir, que los motivos no son independientes de la estancia. En general, los resultados de los motivos pull no parecen ser tan importantes o mencionados como los motivos push y a nivel comparativo, los turistas de short break están más motivados por factores pull, y los del grupo long break por elementos push.

\section{RESULTADOS}

Los datos han sido estudiados utilizando modelos de ecuaciones estructurales, análisis multigrupo para el modelo de la Figura 1. El modelo multigrupo de ecuaciones estructurales fue analizado por dos grupos: Short break y long break. Los resultados y el grado de cumplimiento de las diferentes hipótesis planteadas se detallan en la Tabla 5.

\section{Tabla 5}

RESULTADOS DEL MODELO ESTRUCTURAL

\begin{tabular}{llll}
\hline & & \multicolumn{1}{c}{ Short break } & \multicolumn{1}{c}{ Long break } \\
\hline $\mathrm{H}_{1}$ & Motivación-Satisfacción & -.006 & -.017 \\
$\mathrm{H}_{2}$ & Motivación-Repetitividad & $.427 * * *$ & $.369 * * *$ \\
$\mathrm{H}_{3}$ & Satisfacción-Repetitividad & $-.123 * * *$ & $-.079 *$ \\
$\mathrm{H}_{4}$ & Satisfacción-Recomendación & $.080 * * *$ & $.048 * * *$ \\
$\mathrm{H}_{5}$ & Motivación-Imagen & $.238^{* * *}$ & $.065^{* *}$ \\
$\mathrm{H}_{6}$ & Imagen-Repetitividad & $.319 * * *$ & $.213 * *$ \\
$\mathrm{H}_{7}$ & Imagen-Recomendación & $.364 * * *$ & $.312 * * *$ \\
$\mathrm{H}_{8}$ & Imagen-Satisfacción & $.968 * * *$ & $1.061 * * *$ \\
$\mathrm{H}_{9}$ & Repetitividad-Recomendación & $-.009 *$ & $-.029 * * *$ \\
\hline$* * * p<.001 ; * * p<.01 ; * p<.05$ & &
\end{tabular}

Para la evaluación del ajuste del modelo se aplican diferentes medidas de bondad de ajuste. El SRMR - Standardized Root Mean Square Residual y el RMSEA - Root Mean Square Error of Approximation, se considera un ajuste aceptable si los valores de SRMR son 0.09 o menores y RMSEA de 0.06 (Chen, 2007). Además, se utiliza como índice de 
ajuste incremental, el (CFI - Comparative Fit Index) y el índice de Tucker-Lewis (TLI Tucker - Lewis Index) para calcular las mejoras sobre los modelos de la competencia. Se consideran valores superiores a 0.90 para estos dos índices como indicadores del modelo aceptable (Chen, 2007). La evaluación de esta medida está combinada con el examen de los MI - Modification Index, EPC - Expeted Parameter Change y la potencia de la prueba para una posible especificación errónea (Saris, Satorra y van der Veld, 2009).

Los ajustes del modelo multigrupo de la Tabla 5 son: Chi-square $=94.615 ; 12 \mathrm{df}$; p-value=.000; RMSEA = .037 con 90\% intervalo de confianza (.030; .044); CFI=.985; TLI=.962; SRMR=.019. Por lo tanto, el ajuste del modelo es aceptable según los índices de ajuste y está correctamente especificado (Saris et al., 2009).

En cuanto a los resultados de las hipótesis cabe destacar:

Primero, en la hipótesis $\mathrm{H}_{1}$ el efecto de motivación en la satisfacción del turista no es significativo, por lo que la motivación, push o pull, no influye en la satisfacción. Es decir, la satisfacción del turista en el destino es igual para los turistas que viajan por motivos push, que para los que lo hacen por motivos pull. Este efecto no es significativo para ninguno de los grupos, ni short break, ni long break.

Segundo, en la hipótesis $\mathrm{H}_{2}$ el efecto de motivación en la frecuencia de repetitividad es estadísticamente significativo y positivo. De los resultados obtenidos de esta hipótesis destaca que los turistas que viajan al destino por motivos pull, tienen una mayor repetitividad. Este efecto se mantiene significativo para las categorías de la duración de la estancia, aunque el efecto es más fuerte (.427) para los turistas de short break.

Tercero, en la hipótesis $\mathrm{H}_{3}$ la satisfacción de turista tiene un efecto negativo en la repetitividad. Por lo que, a mayor satisfacción general del turista, menor repetitividad. En este sentido resulta destacable que aquellos turistas que están más satisfechos, repiten menos.

Cuarto, en la hipótesis $\mathrm{H}_{4}$ el nivel de satisfacción tiene un efecto positivo significativo con el nivel de recomendación. Los resultados muestran que los turistas con una valoración de satisfacción mayor, tienen un nivel más alto de recomendación personal. Esta hipótesis es aceptable, tanto para los short break, como para los long break. Aunque si comparamos los efectos según la duración de la estancia, existe un efecto mayor para los short break, que para los long break.

Quinto, en la hipótesis $\mathrm{H}_{5}$ el efecto de la motivación en la imagen es positivo y significativo. Esto implica que los turistas que tienen una motivación pull, tienen una valoración más elevada de la imagen percibida del destino. Comparativamente se observa que los resultados de los turistas de short break, son más elevados (.238), que los turistas de long break (.065).

Sexto, en la hipótesis $\mathrm{H}_{6}$ el efecto de la imagen percibida sobre la repetitividad es estadísticamente significativo y positivo. Los resultados prueban que un nivel más alto de imagen percibida, lleva a una mayor repetitividad. Comparando los resultados, se observa que este efecto es significativo en ambos grupos, aunque el efecto es mayor para los short break (.319), que para los long break (.213).

Séptimo, en la hipótesis $\mathrm{H}_{7}$ el efecto de la imagen percibida sobre la recomendación es estadísticamente significativo y positivo, lo que significa que un nivel más alto de imagen percibida, lleva a una mayor intención de recomendación por parte de los turis- 
tas. Si se comparan los resultados de la duración de la estancia, el efecto es para ambos significativo, aunque es mayor para los short break (.364), que para los long break (.312).

Octavo, en la hipótesis $\mathrm{H}_{8}$ el efecto de la imagen percibida sobre la satisfacción general del turista es estadísticamente significativo y positivo, lo que implica que cuanto mayor es el nivel de imagen percibida, más satisfacción sobre el destino. Comparativamente se observa que este efecto es siempre significativo, aunque es mayor para los turistas de long break, que para los turistas de short break.

Finalmente, en la hipótesis $\mathrm{H}_{9}$, el efecto de la repetitividad en la recomendación es estadísticamente significativo y negativo. Este resultado implica que los turistas que repiten menos, son los que más recomiendan. En los resultados comparativos por grupos, se puede observar que la relación entre repetitividad y recomendación es mayor para los turistas de long break, que para el resto.

De las nueve hipótesis iniciales, tan sólo una, la $\mathrm{H}_{1}$ no es aceptada, por cuanto las relaciones entre motivación y satisfacción no son significativas, ni para los turistas de short break, ni para los de long break. Los turistas de short break tienen un efecto o relación más intensa para las hipótesis $\mathrm{H}_{2}, \mathrm{H}_{3}, \mathrm{H}_{4}, \mathrm{H}_{5}, \mathrm{H}_{6}$ y $\mathrm{H}_{7}$, mientras que para los turistas de long break la relación entre constructos es más fuerte en las hipótesis $\mathrm{H}_{8}$ y $\mathrm{H}_{9}$.

\section{CONCLUSIONES, IMPLICACIONES Y LIMITACIONES}

El presente estudio investiga las relaciones entre motivación, satisfacción, imagen, repetitividad y recomendación de destino, para cada grupo de la variable moderadora, duración de la estancia, short break y long break. Uno de los objetivos es interpretar las diferentes hipótesis según los subsegmentos de short y long break, con necesidades y características diferenciadas. Esta caracterización de segmentos tiene implicaciones significativas, tanto en la planificación, como en la gestión turística de Barcelona, y en especial en aquellas estrategias de marketing y desarrollo de productos turísticos del destino.

Las principales diferencias encontradas entre el grupo de los viajes de short break y long break son las siguientes. Para el grupo de los short break, las relaciones entre motivación y repetitividad $\left(\mathrm{H}_{2}\right)$, entre satisfacción y recomendación $\left(\mathrm{H}_{4}\right)$, entre motivación e imagen $\left(\mathrm{H}_{5}\right)$, e imagen y repetitividad $\left(\mathrm{H}_{6}\right)$, entre imagen y recomendación $\left(\mathrm{H}_{7}\right)$, y entre imagen y satisfacción $\left(\mathrm{H}_{8}\right)$ son hipótesis aceptadas con un alto grado de cumplimiento. Los responsables del destino deberían prestar especial atención a los aspectos de imagen del destino, ya que inciden directamente en la repetitividad, recomendación y satisfacción de los turistas de ocio. Los factores de motivación push y pull tienen efecto directo sobre la repetitividad y la imagen del destino Barcelona. De la misma manera, entre la satisfacción y la recomendación también se prueban relaciones causales positivas importantes, por lo que la mejora de la satisfacción de las experiencias de los turistas, aumenta su recomendación del destino.

En los turistas de short break, los factores de motivación push y pull, no son determinantes para predecir la satisfacción general del turista $\left(\mathrm{H}_{1}\right)$, por lo que se entiende que están igual de satisfechos aquellos que vienen por motivaciones propias de la ciudad o por motivaciones personales. En cuanto a la repetitividad destaca que existe una 
relación negativa de baja intensidad con la recomendación $\left(\mathrm{H}_{9}\right)$, por lo que de manera moderada el aumento de la repetitividad, disminuye la recomendación que hacen los turistas del destino. En este caso podría considerarse que el fomento de las estancias cortas, mejoraría la lealtad de los turistas, en el sentido de recomendación y repetitividad. Este resultado constituye un gran elemento a destacar, ya que en contra de lo que podríamos pensar, los turistas repetidores, recomiendan menos. Este fenómeno puede deberse a factores como el deseo de "quiero explicártelo" en los turistas que visitan el destino por primera vez, así como la disminución de recomendación activa de los visitantes repetidores al perder el efecto "novedad".

Para acabar con este grupo, resulta significativo que los turistas más satisfechos, son los menos repetidores $\left(\mathrm{H}_{3}\right)$. Este resultado constituye uno de los más destacables y a la vez inesperados del estudio, ya que contradice la creencia popular y estudios anteriores (Chi y Qu, 2008). La explicación de este fenómeno puede deberse a que el aumento del conocimiento del destino se traduce en una reducción de satisfacción al disminuir las expectativas creadas y al entrar en contacto con la ciudad real.

Las grandes características específicas de los turistas de long break son la importante diferencia en intensidad en la relación negativa entre la satisfacción y la repetitividad $\left(\mathrm{H}_{3}\right)$. Por lo tanto, los turistas con una satisfacción más alta, son lo que repiten menos veces. De igual forma, en la relación entre repetitividad y recomendación, a mayor repetitividad, menor recomendación $\left(\mathrm{H}_{9}\right)$. Finalmente, las dos últimas características diferenciales de los long break son: la imagen tiene relación sobre la repetitividad $\left(\mathrm{H}_{6}\right)$, aunque con menor grado de intensidad que los short break, además de un menor grado de intensidad en la relación entre motivación e imagen $\left(\mathrm{H}_{5}\right)$.

El estudio presenta algunas limitaciones que deben tenerse en consideración. En primer lugar, los resultados corresponden con el caso de análisis de la ciudad de Barcelona. Para poder generalizarlos se debería aplicar el mismo estudio en varias ciudades turísticas. La aplicación del modelo propuesto a otros destinos puede proporcionar evidencias para extender y generalizar las relaciones causales del modelo. En segundo lugar, este estudio utiliza datos secundarios de una encuesta existente realizada por la DMO de Barcelona, Turisme de Barcelona. Es decir, tanto las preguntas, como las variables de estudio plantean limitaciones, que permitirían un desarrollo específico para obtener datos más precisos. Aun así, los datos confirman anteriores estudios parciales en la misma dirección mostrando su validez. En tercer lugar, tanto la satisfacción, como la repetitividad y la recomendación, fueron medidas mediante una única cuestión/variable y la imagen mediante dos. El uso de escalas de medida multielemento en futuros estudios incrementaría la interpretación y predicción de la satisfacción, la motivación, la imagen, y la recomendación. Los datos de la investigación corresponden al período 2009-2011, habiendo transcurrido cierto tiempo desde la obtención de los mismo. Aun así, la realidad de la demanda turística de la ciudad no ha cambiado sustancialmente en los últimos años. Por lo tanto, para futuras investigaciones se podrían corregir algunas de las limitaciones detectadas en las variables de la encuesta, así como, realizar el mismo estudio, con los mismos criterios, variables y encuestas para el grupo de los turistas de negocios, y comprobar las diferencias existentes con los turistas de short break y long break de la ciudad de Barcelona. 


\section{ANEXO}

Tabla A1

\section{ANÁLISIS DESCRIPTIVO DE LAS VARIABLES USADAS EN EL MODELO ESTRUCTURAL}

\begin{tabular}{lcccc}
\hline & \multicolumn{2}{c}{ Short break } & \multicolumn{2}{c}{ Long break } \\
\hline & Mean & $\begin{array}{c}\text { Standard } \\
\text { deviation }\end{array}$ & Mean & $\begin{array}{c}\text { Standard } \\
\text { deviation }\end{array}$ \\
\hline Motivación pull & 0.176 & .381 & 0.158 & .365 \\
Amplia oferta de puntos de interés & 4.483 & .554 & 4.429 & .578 \\
Tiene una vida cultural muy rica & 4.432 & .563 & 4.404 & .580 \\
"Satisfacción general" & 8.059 & .911 & 8.085 & .958 \\
"Repetitividad" & 0.732 & 1.623 & 0.711 & 1.541 \\
"Recomendación" & 4.819 & .452 & 4.805 & .434 \\
\hline
\end{tabular}

\section{BIBLIOGRAFÍA}

ALEGRE, J. y GARAU, J. (2010): «Tourist Satisfaction and Dissatisfaction», Annals of Tourism Research, vol. 37, $\mathrm{n}^{\circ}$ 1, pp. 52-73.

ALEGRE, J. y POU, L. (2006): «The length of stay in the demand for tourism», Tourism Management, vol. 27, nº 6, pp. 1343-1355.

ANDERSON, E.W. (1998): «Customer Satisfaction and Word of Mouth», Journal of Service Research, vol. 1, n ${ }^{\circ}$ 1, pp. 5-17.

ANDRÉ ROMERO, M.E. (1999): Turismo urbano y la segmentación motivacional: aplicación econométrica a la ciudad de Barcelona, Ed. Universitat de Barcelona Disponible en http://www.tesisenred.net/handle/10803/1462

ASSAF, A.G., PESTANA BARROS, C. y GIL-ALANA, L.A. (2010): «Persistence in the Short- and Long-Term Tourist Arrivals to Australia», Journal of Travel Research, vol. 50, no 2, pp. 213-229.

BALOGLU, S. y MCCLEARY, K.W. (1999): «A model of destination image formation», Annals of Tourism Research, vol. 26, n 4, pp. 868-897.

BALOGLU, S. y UYSAL, M. (1996): «Market segments of push and pull motivations: a canonical correlation approach», International Journal of Contemporary Hospitality Management, vol. 8, $\mathrm{n}^{\mathrm{o}}$ 3, pp. 32-38.

BARROS, C. P., CORREIA, A. y CROUCH, G. (2008): «Determinants of the Length of Stay in Latin American Tourism Destinations», Tourism Analysis, vol. 13, n 4, pp. 329-340.

BERNINI, C., y CAGNONE, S. (2014): «Analysing tourist satisfaction at a mature and multi-product destination», Current Issues in Tourism, vol. 17, $\mathrm{n}^{\circ}$ 1, pp. 1-20.

BIGNÉ ALCAÑIZ, E., SÁNCHEZ, M.I. y SÁNCHEZ, J. (2001): «Tourism image, evaluation variables and after purchase behaviour: inter-relationship», Tourism Management, vol. 22, n 6, pp. 607-616. 
BUHALIS, D. (2001): The new tourist and consumer. In C. Wahab, S.; Cooper (Ed.), Tourism in the age of Globalisation. London: Routledge.

CAMPO, S., y YAGÜE, M. J. (2008) : «Effects of price on tourist satisfaction», Tourism Economics, vol. 14, n 3, pp. 657-661.

CAMPRUBÍ, R., GUIA, J. y COMAS, J. (2009): «La formación de la imagen turística inducida: Un modelo conceptual», Pasos, Revista de Turismo Y Patrimonio Cultural, vol. 7, $\mathrm{n}^{\mathrm{o}} 2$, pp. 255-270.

CASELlAS, A., DOT JUTGLA, E. y PALLARES-BARBERA, M. (2010): «Creación de imagen, visibilidad y turismo como estrategias de crecimiento económico de la ciudad», Finisterra, vol. 45, no 90, pp. 153-172.

CHARTERINA, J. y APARICIO, G. (2015): «A comparison of antecedents of satisfaction in city break traveling», Revista Española de Investigación En Marketing ESIC, vol. $19, \mathrm{n}^{\circ} 2$, pp. 71-82.

CHEN, C.-F. y PHOU, S. (2013): «A closer look at destination: Image, personality, relationship and loyalty», Tourism Management, vol. 36, June 2013, pp. 269-278.

CHEN, F.F. (2007): «Sensitivity of Goodness of Fit Indexes to Lack of Measurement Invariance», Structural Equation Modeling: A Multidisciplinary Journal, vol.14, $\mathrm{n}^{\circ}$ 3 , pp. 464-504.

CHENG, S., LAM, T. y HSU, C.H.C. (2006): «Negative Word-of-Mouth Communication Intention: An Application of the Theory of Planned Behavior», Journal of Hospitality \& Tourism Research, vol. 30, $\mathrm{n}^{\mathrm{o}} 1$, pp. 95-116.

CHI, C.G.-Q. y QU, H. (2008): «Examining the structural relationships of destination image, tourist satisfaction and destination loyalty: An integrated approach», Tourism Management, vol. 29, $\mathrm{n}^{\circ}$ 4, 624-636.

CHON, K.-S. (1991): «Tourism destination image modification process: Marketing implications», Tourism Management, vol. 12, n 1, pp. 68-72.

CROMPTON, J.L. (1979): «Motivations for pleasure vacation», Annals of Tourism Research, vol. 6, nº 4, pp. 408-424

CROMPTON, J L. y ANKOMAH, P.K. (1993): «Choice set propositions in destination decisions», Annals of Tourism Research, vol. 20, nº 3, pp. 461-476.

DA COSTA MENDES, J., DO VALLE, P.O., GUERREIRO, M. M. y SILVA, J. (2010): «The tourist experience: Exploring the relationship between tourist satisfaction and destination loyalty», Turizam: znanstveno-stručni časopis, vol. 58, n 2, pp. 111-126.

DAVIES, B. (1990): «The economics of short breaks», International Journal of Hospitality Management, vol. 9, $\mathrm{n}^{\circ}$ 2, pp. 103-106.

DEGEN, M. y GARCÍA, M. (2012): «The Transformation of the "Barcelona Model": An Analysis of Culture, Urban Regeneration and Governance», International Journal of Urban and Regional Research, vol. 36, n 5, pp. 1022-1038.

DOWNWARD, P. y LUMSDON, L. (2003): «Beyond the demand for day-visits: an analysis of visitor spending», Tourism Economics, vol. 9, n 1, pp. 67-76.

DUNNE, G., FLANAGAN, S. y BUCKLEY, J. (2010): «Towards an understanding of international city break travel», International Journal of Tourism Research, vol 12, $\mathrm{n}^{\circ}$ 5, pp. 409-417. 
DUNNE, G., FLANAGAN, S, y BUCKLEY, J. (2011): «Towards a decision making model for city break travel», International Journal of Culture, Tourism and Hospitality Research, vol. 5, $\mathrm{n}^{\circ} 2$, pp. 158-172.

ECM. (2013). ECM Benchmarking Report - 9th edition: Figures from more than 110. Dijon. EDGAR, D.A. (1997): «Capacity management in the short break market», International Journal of Contemporary Hospitality Management, vol. 9, n 2, pp. 55-59.

FAKEYE, P.C, y CROMPTON, J.L. (1991): «Image Differences between Prospective, First-Time, and Repeat Visitors to the Lower Rio Grande Valley», Journal of Travel Research, vol. 30, n 2, pp. 10-16.

FLAVIAN, C., MARTÍNEZ, E. y POLO, Y. (2001): «Loyalty to grocery stores in the Spanish market of the 1990s», Journal of Retailing and Consumer Services, vol. 8, $\mathrm{n}^{\mathrm{o}} 2$, pp. 85-93.

FORGAS-COLL, S., PALAU-SAUMELL, R., SÁNCHEZ-GARCÍA, J. y CALLARISAFIOL, L.J. (2012): «Urban destination loyalty drivers and cross-national moderator effects: The case of Barcelona», Tourism Management, vol. 33, n 6, pp. 1309-1320.

FYALL, A., CALLOD, C. y EDWARDS, B. (2003): «Relationship Marketing», Annals of Tourism Research, vol. 30, no 3, pp. 644-659.

GALÍ ESPELT, N. y DONAIRE BENITO, J.A. (2005): «The social construction of the image of Girona: a methodological approach», Tourism Management, vol. 26, $\mathrm{n}^{\circ} 5$, pp. 777-785.

GALLARZA, M., GIL-SAURA, I. y CALDERÓ-GARCÍA, H. (2002): «Destination Image Towards a conceptual framework», Annals of Tourism Research, vol. 29, $\mathrm{n}^{\circ} 1$, pp. 56-78.

GARTNER, W.C. (1989): «Tourism Image: Attribute Measurement of State Tourism Products Using Multidimensional Scaling Techniques», Journal of Travel Research, vol. 28, no 2 , pp. 16-20.

GARTNER, W.C. (1994): «Image Formation Process. Journal of Travel \& Tourism Marketing, vol. 2, no 2-3, pp. 191-216.

GOVERS, R., y GO, F. (2004): «Cultural identities constructed, imagined and experienced: a 3-gap tourism destination image model», Tourism (13327461). Vol. 52, $\mathrm{n}^{\circ} 2$, pp. 165-182.

HUNT, J. D. (1971) : Image: a factor in tourism, Colorado State Univeristy.

ISO-AHOLA, S.E. y ALLEN, J.R. (1982): «The Dynamics of Leisure Motivation: The Effects of Outcome on Leisure Needs», Research Quarterly for Exercise and Sport, vol. 53, n ${ }^{\circ} 2$, pp. 141-149.

KOTLER, P., HAIDER, D.H. y REIN, I. (1994): Mercadotecnia de localidades. México: Diana.

KOZAK, M. (2001): «Repeaters' behavior at two distinct destinations», Annals of Tourism Research, vol. 28, n 3, pp. 784-807.

KOZAK, M., BIGNE, E. y ANDREU, L. (2005): «Satisfaction and Destination Loyalty», Journal of Quality Assurance in Hospitality \& Tourism, vol. 5, n ${ }^{\circ}$ 1, pp. 43-59.

KOZAK, M., y RIMMINGTON, M. (2000): «Tourist Satisfaction with Mallorca, Spain, as an Off-Season Holiday Destination», Journal of Travel Research, vol. 38, $\mathrm{n}^{\circ} 3$, pp. 260-269. 
LI, M. y CAI, L.A. (2011): «The Effects of Personal Values on Travel Motivation and Behavioral Intention», Journal of Travel Research, vol. 51, n 4, pp. 473-487.

LIN, Y., KERSTETTER, D., NAWIJN, J. y MITAS, O. (2014): «Changes in emotions and their interactions with personality in a vacation context», Tourism Management, vol. 40, pp. 416-424.

LUPTON, R.A. y COURT, B. (1997): «Customer Portfolio Development: Modeling Destination Adopters, Inactives, and Rejecters», Journal of Travel Research, vol. 36, $\mathrm{n}^{\mathrm{o}} 1$, pp. 35-43.

MARTÍNEZ-GARCIA, E. y RAYA, J.M. (2008): «Length of stay for low-cost tourism», Tourism Management, vol. 29, $n^{\circ}$ 6, pp. 1064-1075.

MARTÍNEZ-RUIZ, M.P., GARAU-VADELL, J.B. y CAMPO-MARTÍNEZ, S. (2010): «Factors influencing repeat visits to a destination: The influence of group composition», Tourism Management, vol. 31, nº 6, pp. 862-870.

MELEDDU, M., PACI, R. y PULINA, M. (2015): «Repeated behaviour and destination loyalty», Tourism Management, vol. 50, pp. 159-171.

MOHAMMAD, B.A.M.A.-H. y SOM, A.P.M. (2010): «An Analysis of Push and Pull Travel Motivations of Foreign Tourists to Jordan», International Journal of Business and Management, vol. 5, $\mathrm{n}^{\circ}$ 12, pp. 41-50.

MOLINA COLLADO, A. (2007): «Segmentación de la demanda turistica: un análisis aplicado a un destino de turismo cultural», Revista de Análisis Turistico, vol. 4, n 4 , pp. 36-48.

MONIZ, A. I. (2012): «A dynamic analysis of repeat visitors», Tourism Economics, vol. 18 , pp. 505-517).

MURPHY, P., NIININEN, O. y SANDERS, D. (2010): Short-break holidays-a competitive destination strategy, Australia, CRC for Sustainable Tourism Pty Ltd. Disponible en http://www.crctourism.com.au/wms/upload/resources/110019\%20Murphy\%20 Short\%20Breaks\%20WEB\%20\%281\%29.pdf

OFICINA PET BCN2015. (2009): Quantificació del Nombre de visitants de la ciutat de Barcelona, Barcelona.

OLIVER, R. (1999): «Whence Consumer Loyalty? The Journal of Marketing, vol. 63, pp. 33-44.

OLIVER, R.L. (1980): «A cognitive model of the antecedents and consequences of satisfaction decisions», Journal of Marketing Research, vol. 17, $\mathrm{n}^{\circ}$ 4, pp. 460-469.

OLIVER, R. L. y SWAN, J. (1989): «Consumer perceptions of interpersonal equity and satisfaction in transactions: a field survey approach», The Journal of Marketing, vol. 53, nº 2, pp. 21-35.

OPPERMANN, M. (1995): «Travel life cycle», Annals of Tourism Research, vol. 22, $\mathrm{n}^{\circ}$ 3 , pp. 535-552.

OPPERMANN, M. (2000): «Tourism Destination Loyalty», Journal of Travel Research, vol. 39, no 1 , pp. 78-84.

PALAU-SAUMELL, R., FORGAS-COLL, S., SANCHEZ-GARCIA, J. y PRATS-PLANAGUMA, L. (2012): «Tourist Behavior Intentions and the Moderator Effect of Knowledge of UNESCO World Heritage Sites: The Case of La Sagrada Familia», Journal of Travel Research, vol. 52, n 3, pp. 364-376. 
PALOU RUBIO, S. (2011): Barcelona, destinació turística. Promoció pública, turismes, imatges i ciutat (1888-2010), UB Universitat de Barcelona. Disponible en http://www. tdx.cat/handle/10803/21771

PEYPOCH, N., RANDRIAMBOARISON, R., RASOAMANANJARA, F. y SOLONANDRASANA, B. (2012): «The length of stay of tourists in Madagascar», Tourism Management, vol. 33, $\mathrm{n}^{\circ}$ 5, pp. 1230-1235.

PHILLIPS, W.J., WOLFE, K., HODUR, N. y LEISTRITZ, F. L. (2013): «Tourist Word of Mouth and Revisit Intentions to Rural Tourism Destinations: a Case of North Dakota, USA», International Journal of Tourism Research, vol. 15, n 1, pp. 93-104.

PIKE, S. (2009): «Destination brand positions of a competitive set of near-home destinations», Tourism Management, vol. 30, $\mathrm{n}^{\circ}$ 6, pp. 857-866.

RAJ, A. (2007): «The New Age of Tourism and the New Tourist». Disponible en http:// www.indianmba.com/Faculty_Column/FC565/fc565.html.

SARIS, W. E., SATORRA, A. y VAN DER VELD, W. M. (2009): «Testing Structural Equation Models or Detection of Misspecifications?», Structural Equation Modeling: A Multidisciplinary Journal, vol. 16, $\mathrm{n}^{\circ}$ 4, pp. 561-582.

SASSEN, S. (2007): «El reposicionamiento de las ciudades y regiones urbanas en una economía global: ampliando las opciones de políticas y gobernanza», EURE (Santiago), vol. 33, n 100, pp. 9-34.

SIRAKAYA-TURK, E., SONMEZ, S. F. y CHOI, H.-S. (2001): «Do destination images really matter? Predicting destination choices of student travellers», Journal of Vacation Marketing, vol. 7, n 2, pp. 125-142.

SOM, A.P.M., MARZUKI, A., YOUSEFI, M., y ABUKHALIFEH, A. N. (2012): «Factors influencing visitors' revisit behavioral Intentions: A case study of Sabah, Malaysia», International Journal of marketing studies, vol. 4, $\mathrm{n}^{\circ}$ 4, pp. 39-50.

STERN, E. y KRAKOVER, S. (1993): «The formation of a Composite Urban Image», Geographical Analysis, vol. 25, n 2, pp. 130-146.

TSIOTSOU, R. y VASIOTI, E. (2006): «Satisfaction», Journal of Travel \& Tourism Marketing, vol. 20, $\mathrm{n}^{\circ} 1$, pp. 61-73.

TURISME DE BARCELONA. (2014): Estadísticas de Turismo en Barcelona y comarcas, Barcelona, Turisme de Barcelona.

UYSAL, M. y JUROWSKI, C. (1994): «Testing the push and pull factors», Annals of Tourism Research, vol. 21, no 4, pp. 844-846.

VALLS, J., BANCHINI, S., FALCÓN, L. y VALLS TUÑON, G. (2013): «Repositioning of Barcelona's Image in the Light of a Redefinition of the Urban Tourism Planning Model», Pasos, Revista de Turismo Y Patrimonio Cultural, vol. 11, n 1, pp. 89-105.

YOON, Y. y UYSAL, M. (2005): «An examination of the effects of motivation and satisfaction on destination loyalty: a structural model», Tourism Management, vol. 26, $\mathrm{n}^{\mathrm{o}} 1$, pp. 45-56.

YUAN, S. y MCDONALD, C. (1990): «Motivational Determinates Of International Pleasure Time», Journal of Travel Research, vol. 29, nº 1, pp. 42-44.

ZHANG, H., FU, X., CAI, L. A. y LU, L. (2014): «Destination image and tourist loyalty: A meta-analysis», Tourism Management, vol. 40, pp. 213-223. 